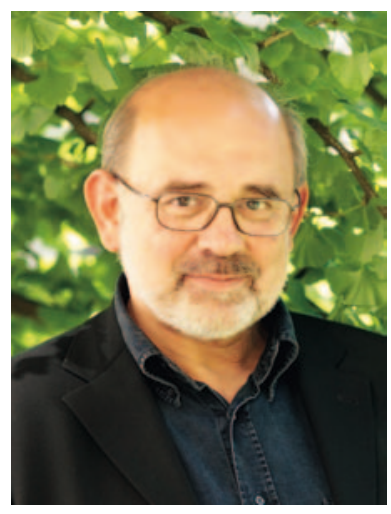

Prof. Dr. med. Reinhard Saller, Zürich

\section{Phytotherapie und phytotherapeutischer Wirkstoff}

Die Phytotherapie ist ein hochdifferenzierter und gleichzeitig mannigfaltiger Teil der modernen Medizin. Phytotherapeutika sind Arznei- und Heilmittel, die aufgrund der Geschichte der Heilpflanzen, aus denen sie entwickelt werden, unterschiedliche Medizinkonzepte, diverse Betrachtungsweisen von Gesundheit, Krankheit und Genesung, aber auch unterschiedliche, häufig komplementäre Behandlungsansätze mit sich führen. Sie können einerseits Arzneimittel im Rahmen moderner schulmedizinischer Krankheitskonzepte und andererseits verschiedener traditioneller wie moderner komplementärmedizinischer Richtungen sein. Dementsprechend besitzen viele Phytotherapeutika eine hohe Anschlussfähigkeit in der gesamten modernen Medizin.

Unabhängig von ihrem konzeptionellen Bezug besitzen Phytotherapeutika einige charakteristische Eigenschaften, die sie von anderen Arzneimitteln unterscheiden. Dazu gehört der genuine Vielstoffcharakter des phytotherapeutischen Wirkstoffes eines Mono- wie auch eines Kombinationsphytotherapeutikums. Die einzelnen Komponenten dieser Wirkstoffe liegen zumeist in sehr niedrigen Konzentrationen vor. In solchen Konzentrationen würden die Komponenten bei alleiniger Anwendung zumeist keine mit modernen klinischen Studieninstrumenten fassbaren Wirkungen besitzen. Erst durch die gleichzeitige Anwesenheit der anderen Komponenten und den vielfältigen Möglichkeiten für Interaktionen innerhalb des Wirkstoffes erhalten sie ihre Wirksamkeit. Bei solchen Arzneimitteln kann angenommen werden, dass ein solcher Wirkstoff eine Reihe von Wirkmechanismen besitzt, die voneinander unabhängig sind (Pleiotropie von Phytotherapeutika). Diese Pleiotropie ermöglicht eine Reihe von Vorteilen. Fällt z.B. ein physiologischer Weg im Organismus aus, auf dem die Wirkungen eines Arzneimittels basieren, so können pleiotrope Arzneimittel dennoch wirksam sein, da der Wirkstoff auch andere Stoffwechselwege beeinflussen kann. So wirken eine Reihe antiinflammatorischer Phytotherapeutika nicht nur über eine Hemmung von Cyclooxygenasen, sondern auch über eine Hemmung von Lipoxygenasen und weiteren Mechanismen (z.B. verschiedene Zytokinine, $\mathrm{NF}_{\mathrm{kappaB}}$ ). Allerdings werden Stoffwechselwege aufgrund des beschriebenen Vielstoffcharakters z.B. nicht vollständig gehemmt oder stimuliert. Insgesamt lassen sich phytotherapeutische Wirkstoffe auch mit Kriterien einer Art von Netzwerken charakterisieren.

Phytotherapeutische Wirkstoffe besitzen aufgrund ihrer Charakteristika zumeist ein in qualitativer Hinsicht breiteres Wirkungsspektrum als viele Monosubstanzen (genuine Multi-Target-Arzneimittel). Dies weist darauf hin, dass mit einem kompetent ausgewählten Phytotherapeutikum mehrere Behandlungsanlässe bei einem Patienten gleichzeitig behandelt werden können. Dies könnte z.B. zu einer Abnahme pharmakotherapeutischer Polypragmasien führen. Ebenso könnten sich daher Phytotherapeutika auch zur Behandlung komplexer funktioneller Krankheiten eignen. Gerade auch im Zusammenhang z.B. mit einer häufigen Polymorbidität bei älteren Patienten könnten sich Phytotherapeutika als zentrale Bestandteile von möglichst einfachen Behandlungskonzepten erweisen.

Vielstoffcharakter und Pleiotropie könnten auch dazu beitragen, dass eine Reihe von Phytotherapeutika zu einer relativ niedrigen Inzidenz unerwünschter Wirkungen zu führen scheinen.

Der Vielstoffcharakter phytotherapeutischer Wirkstoffe weist darauf hin, dass zumeist von Daten einer Einzelkomponente nicht hinreichend auf Wirkungen und Wirksamkeit des Vielstoffgemisches geschlossen werden kann. Um diese abschätzen zu können, muss der jeweils therapeutisch verwendete Wirkstoff selbst untersucht werden. Eine gewisse Ausnahme könnten z.B. Zubereitungen mit Curcuma oder Kampfer darstellen, bei denen im phytotherapeutischen Wirkstoff Einzelkomponenten bzw. wenige Einzelkomponenten dominieren.

\section{KARGER}

Fax +497614520714 Information@Karger.d www.karger.com (c) 2010 S. Karger GmbH, Freiburg www.karger.com/szg
Prof. Dr. med. Reinhard Saller

Departement Innere Medizin, Institut für Naturheilkunde

Universitätsspital Zürich

Rämistrasse 100, 8091 Zürich, Schweiz

Tel. +41 44255 2460, Fax - 4394

reinhard.saller@usz.ch 
Vergleichbar stellt sich auch der Wirkstoff von phytotherapeutischen Kombinationspräparaten dar, dessen Wirkungen und Wirksamkeit sich nicht zwangsläufig aus der Kenntnis von Einzelkomponenten abschätzen lassen. Einzelkomponenten bei Kombinationspräparaten sind auch die einzelnen Arzneidrogen bzw. deren Zubereitungen, die gemeinsam den Wirkstoff bilden.

Phytotherapeutika lassen sich allerdings therapeutisch sinnvoll auch in traditionellen Zusammenhängen anwenden. Eine solche Möglichkeit sei kurz am Beispiel von Pfefferminze abgehandelt. In einer humoralmedizinischen Betrachtungsweise z.B. besitzt die Pfefferminze warme und trocknende Eigenschaften. Dementsprechend wird sie bevorzugt bei kaltem und feuchtem Magen, Menschen mit kalter und feuchter "complexion» und alten Menschen sowie im Winter und in kalten Gegenden angewendet. Da die Behandlung mit Arznei- und Heilpflanzen - und damit wesentliche Teile der Phytotherapie - kein ärztliches Monopol ist, sondern unter anderem von nichtärztlichen Berufsgruppen ausgeübt wird, greift die «Pfefferminze» auch korrespondierende Denkstile aus anderen Berufsgruppen auf. Die Pfefferminze ist zudem der Prototyp einer Arzneipflanze, die weit verbreitet in der Selbstbehandlung und damit im Kontext patienteneigener Vorstellungen und Kompetenzen angewendet wird (laienmedizinische Vorstellungen).

Arznei- und Heilpflanzen werden zum Teil bereits seit langer Zeit über ihre ursprünglichen Verbreitungsgebiete und den entsprechenden medizinkulturellen Kontext hinaus angewendet. So zeigt auch die weltweite recht unterschiedliche Verwendung der Pfefferminze, dass Phytotherapie bereits lange vor den jetzigen Globalisierungsschüben einerseits kulturell umgrenzte Therapieräume und andererseits globale Verbreitung besass.

Die biologische, geschichtliche, kulturelle und medizinische Vielfalt der Phytotherapeutika lässt sich nicht einfach nur biomedizinisch begreifen, sondern muss unter einem wesentlich umfassenderen biokulturellen Blickwinkel betrachtet werden. Traditionen und Moderne schliessen sich dabei nicht aus.

Prof. Dr. med. Reinhard Saller, Zürich 